\title{
Mutants de Lactococcus lactis résistants à l'acidité
}

\author{
Fabien Rallu, Alexandra Gruss, Emmanuelle Maguin
}

Laboratoire de génétique microbienne, Inra, 78352 Jouy-en Josas cedex, France

\begin{abstract}
Acid-resistant mutants of Lactococcus lactis. In all environments, bacteria are submitted to stress conditions which compromise cell growth and survival. It is now established that bacteria have inducible stress resistance mechanisms which favor bacterial survival under difficult conditions. These resistance mechanisms may be useful for improving strain survival in technological processes. Using $L$. lactis, we selected mutants more resistant to acid stress than the wildtype strain, MG1363. Results of genetic and phenotypic characterization of these mutants established that strain survival is improved compared to MG1363 in a single or up to three different stress conditions, and sometimes by a factor of 2000 . This work shows that in L. lactis, stress responses can overlap and that a mutated strain can be more appropriate than the wild-type to survive abrupt environmental changes. Such strains may have improved properties in industrial processes. (C) Inra/Elsevier, Paris.
\end{abstract}

acid stress / Lactococcus lactis / acid-resistance / multi-stress resistance / transposition

Résumé - Dans tout environnement, les bactéries sont soumises à des stress qui compromettent la multiplication et la survie de l'espèce. Il est désormais établi que les bactéries possèdent des mécanismes inductibles de résistance aux stress qui contribuent à leur survie en conditions défavorables. Pour améliorer la survie des souches en condition technologique, nous avons sélectionné des mutants de Lactococcus lactis plus résistants au stress acide que la souche sauvage, MG1363. La caractérisation génétique et phénotypique de ces mutants révèle que certaines mutations confèrent une survie jusqu'à 2000 fois supérieure à celle de MG1363 dans une, deux ou trois conditions de stress. Cette approche démontre qu'il existe chez $L$. lactis des chevauchements entre les mécanismes de résistance aux stress et que des mutations adéquates peuvent conduire à des souches plus performantes face à de brusques changements environnementaux. De telles souches pourraient améliorer le rendement et la reproductibilité des processus industriels. (C) Inra/Elsevier, Paris.

stress acide / Lactococcus lactis / résistance à l'acidité / résistance multiple / transposition 


\section{INTRODUCTION}

Dans la nature, les conditions de croissance bactérienne sont rarement optimales. Les bactéries subissent des stress imposés par l'environnement tels que la température, l'oxygénation, la salinité... De plus, la croissance bactérienne conduit à des modifications du milieu génératrices de stress : carences nutritionnelles et, dans le cas des bactéries lactiques, acidification du milieu. Il est désormais établi que les bactéries perçoivent les variations du milieu et répondent aux conditions de stress par l'induction de régulons qui permettent à la cellule de réorganiser son métabolisme afin de privilégier sa survie.

Lactococcus lactis est devenu une bactérie lactique modèle, du fait des nombreuses études qui lui ont été consacrées depuis une dizaine d'années. Dans les procédés technologiques, les bactéries rencontrent des contraintes liées au type de production qui constituent des stress. Ces stress affectent la physiologie cellulaire, modifiant les capacités de croissance et la viabilité des bactéries, ce qui peut avoir des conséquences néfastes sur la qualité, le rendement ou la reproductibilité des procédés.

La connaissance des réponses bactériennes aux stress pourrait permettre d'améliorer la résistance des bactéries aux stress technologiques et à terme, d'ajuster la physiologie et la résistance des souches en fonction des traitements technologiques envisagés.

Nous recherchons les gènes impliqués dans les réponses aux stress de $L$. lactis et en particulier les gènes assurant la régulation des mécanismes de résistance. Nous nous sommes intéressés au stress acide dans la mesure où le pouvoir acidifiant et la survie des bactéries en milieu acide sont des critères technologiques importants. De plus, considérant que l'acidité du milieu augmente avec la croissance de la souche, la concentration d'acide lactique pourrait servir de signal d'induction d'une réponse globale au stress, typique de la phase stationnaire.

Plusieurs études ont montré que $L$. lactis s'adapte à l'acidité au fur et à mesure de sa croissance et qu'en condition de carence en glucose, $L$. lactis est plus résistant aux conditions acides $[2,11]$. Ces observations suggèrent qu'il existe chez L. lactis au moins deux mécanismes inductibles (l'un en phase exponentielle, l'autre par la carence en glucose) de résistance au stress acide.

Pour étudier les gènes impliqués dans la régulation de ces réponses aux stress, nous avons réalisé une mutagenèse par transposition [7] et sélectionné des mutants plus résistants au stress acide que la souche parentale. Une vingtaine de mutants ont été caractérisés du point de vue génétique et phénotypique.

\section{RÉSULTATS ET DISCUSSION}

\subsection{Sélection des mutants résistant à l'acidité}

La souche de L. lactis, MG1363 est sensible au $\mathrm{pH}$ acide sur boîte ( $\mathrm{pH} 5$ ou $\mathrm{pH} 5,5)$ à $37^{\circ} \mathrm{C}$. Après mutagenèse par transposition avec le système $\mathrm{pG}+$ host 9 : ISS1 [7], nous avons recherché des mutants capables de former des colonies sur boîte à pH 5 ou 5,5 à $37^{\circ} \mathrm{C}$. Nous avons ainsi isolé une trentaine de clones. La survie de vingt de ces mutants et celle de la souche parentale ont été comparées après un brusque changement de $\mathrm{pH}$ du milieu : les cultures maintenues à $\mathrm{pH}$ supérieur à 6,9 sont transférées en condition acide létale ( $\mathrm{pH} 3$ ou $\mathrm{pH} 3,7$ ). La survie des souches est mesurée par des étalements avant et après transfert en milieu acide. Les 20 mutants testés survivent 6 à 1200 fois mieux à ce stress que la souche parentale, MG1363. 
Ces mutants peuvent être affectés dans un mécanisme de résistance spécifique au stress acide ou dans un mécanisme de résistance à un (ou plusieurs) autre(s) stress qui confère un avantage dans des conditions acides. Pour distinguer ces deux possibilités, le phénotype des mutants a été comparé à celui de la souche parentale dans plusieurs conditions de stress : choc thermique, oxydant ou survie en condition de carence en glucose. Trois classes phénotypiques sont ainsi révélées.

\subsection{Classe 1 : résistance spécifique au stress acide}

Parmi les 20 mutants testés, six s'avèrent plus résistants que la souche initiale uniquement en conditions de stress acide (figure 1). La séquence des gènes inactivés chez ces mutants révèle que les mutants arl3 (acid resistant locus), arl4 et arl8 sont affectés dans des régions d'ADN ne présentant pas d'homologie significative avec les banques de données. Les autres mutants de cette classe correspondent à trois insertions différentes dans un gène de L. lactis homologue à une perméase à glutamine ( $g \ln P$ de Synechocystis sp. [4]).

Les mutations pourraient inactiver un gène dont 1 'expression est néfaste à $\mathrm{pH}$ acide. Cela pourrait être le cas dans les mutants $g \ln P$, puisque l'inactivation de ce gène supprime une activité ATPase consommatrice d'énergie. Alternativement, les mutations pourraient entraîner l'expression constitutive d'un gène (ou d'un régulon) conférant une certaine résistance au stress acide par neutralisation de l'acidité ou protection des fonctions essentielles à la bactérie.

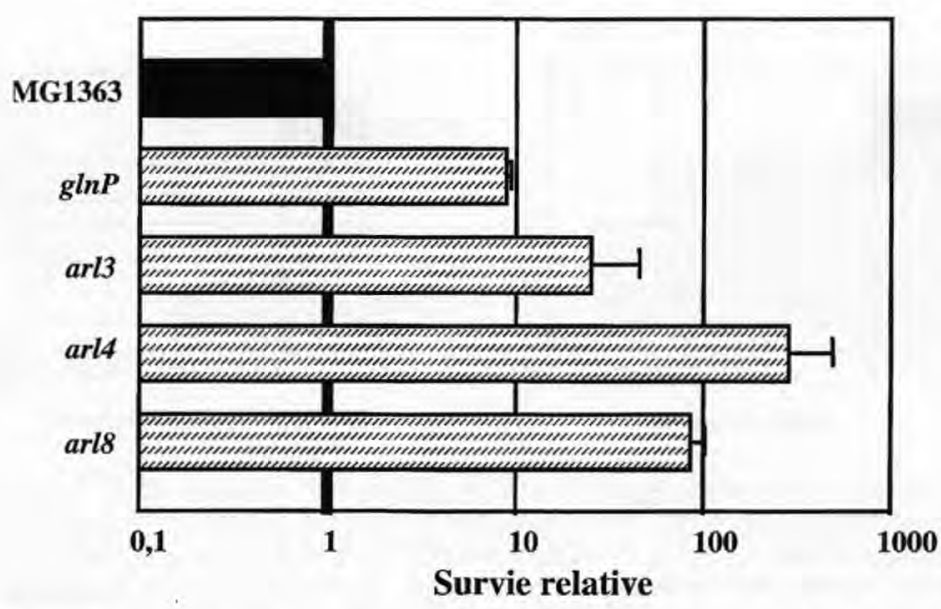

Figure 1. Survie relative des mutants de classe 1 et de MG1363 en conditions acides. Une culture à $\mathrm{pH}$ neutre est transférée à $\mathrm{T} 0$ en condition acide pour un temps $\mathrm{T}$. La survie est mesurée par étalement sur boîte de M17à T0 et T. Le taux de survie est calculé par le rapport entre les deux valeurs (T/T0). La survie relative est le rapport du taux de survie du mutant sur celui de la souche sauvage.

Figure 1. Relative survival of class 1 mutants and MG1363 under acid conditions. A culture at neutral $\mathrm{pH}$ is transferred into acid media at T0. Survival is estimated by plating on M17 before (T0) and after (T) the acid challenge, and the survival rate is calculated as the ratio between the two values (T/T0). The relative survival is the ratio of the survival rate of the mutant and that of the wild-type strain. 


\subsection{Classe 2 : résistance aux stress acide et oxydant}

Cette classe comporte trois mutants dont la caractérisation génétique montre que l'un, pstS, est inactivé dans le transporteur à haute affinité de phosphate [12] et que les deux autres, $\operatorname{arll}$ et $\operatorname{arl} 2$, sont affectés dans une région d'ADN ne présentant aucune homologie avec les bases de données. En condition acide létale, la survie de $p s t S$, de $a r l l$ et de $\operatorname{arl} 2$ augmente respectivement d'un facteur 80,370 et 230 par rapport à MG1363 (figure 2). En présence de peroxyde d'hydrogène, $p s t S$ et arll survivent respectivement 2000 et 800 fois plus que MG1363. Ces résultats suggèrent un certain recouvrement entre les réponses aux stress acide et oxydant. Rappelons ici que chez L. lactis, au moins une protéine induite pendant l'adaptation au stress acide est aussi induite en présence de $\mathrm{H}_{2} \mathrm{O}_{2}$ [2] et que chez Salmonella

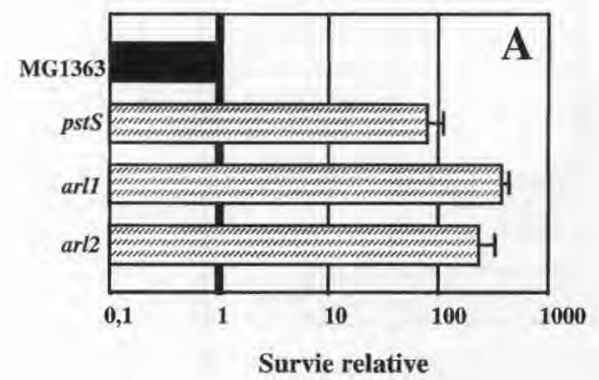

typhimurium, l'adaptation au stress acide augmente la résistance au stress oxydant [1].

\subsection{Classe 3 : résistance aux stress acide, thermique et à la carence en glucose}

Cinq mutants font partie de cette classe. En condition acide, la résistance de ces mutants est de 100 à 1000 fois supérieure à celle de MG1363. De plus, la survie des mutants est aussi améliorée par rapport à celle de MG1363 en conditions de choc thermique ou de carence en carbone à $\mathrm{pH}$ acide ou neutre (figure 3). Notre hypothèse concernant ces mutants est qu'ils sont affectés dans un (ou des) système(s) de régulation de mécanisme(s) de résistance globale aux stress.

Les comparaisons de séquence révèlent que le gène inactivé dans le mutant

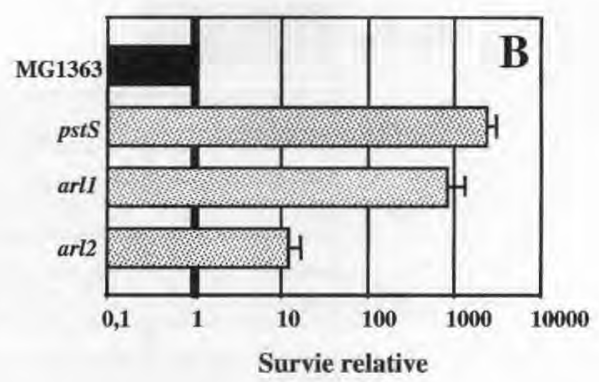

Figure 2. Phénotype des mutants de classe 2. A. Résistance au stress acide. Les cultures en phase exponentielle et à $\mathrm{pH}$ neutre sont transférées à $\mathrm{T} 0$ en milieu à $\mathrm{pH} 3,7$. La survie est mesurée par étalement à $\mathrm{T} 0$ et après $2 \mathrm{~h}$. d'incubation à $\mathrm{pH} 3,7(\mathrm{~T} 2)$. Le rapport $\mathrm{T} 2 / \mathrm{T} 0$ correspond au taux de survie, il est de $710^{-5}$ pour la souche sauvage. La survie relative est calculée par le rapport entre le taux de survie du mutant et celui de la souche sauvage. B. Résistance au stress oxydatif. Le choc oxydatif est réalisé par incubation en présence de $1 \mathrm{mM}$ de $\mathrm{H}_{2} \mathrm{O}_{2}$ pendant $30 \mathrm{~min}$. Les survies relatives des mutants et de la souche sauvage, MG1363, sont calculées comme décrit précédemment.

Figure 2. Phenotype of class 2 mutants. A. Acid-stress survival. Exponential phase cultures grown at neutral $\mathrm{pH}$ were transferred at T0 into acid media ( $\mathrm{pH} 3.7$ ) for $2 \mathrm{~h}(\mathrm{~T} 2)$. Viable counts were measured by plating on M17 at T0 and T2. The survival rate was calculated by the ratio $\mathrm{T} 2 / \mathrm{T} 0$, and corresponds to $710^{-5}$ for the wild-type strain. The relative survival was calculated as the ratio of the survival rate of the mutant and the wild-type. B. Oxidative stress. The oxydative stress was carried out by incubating the cultures with $1 \mathrm{mM} \mathrm{H}_{2} \mathrm{O}_{2}$ during 30 minutes. The survival rates of mutated and wild-type strains were calculated as described above. 

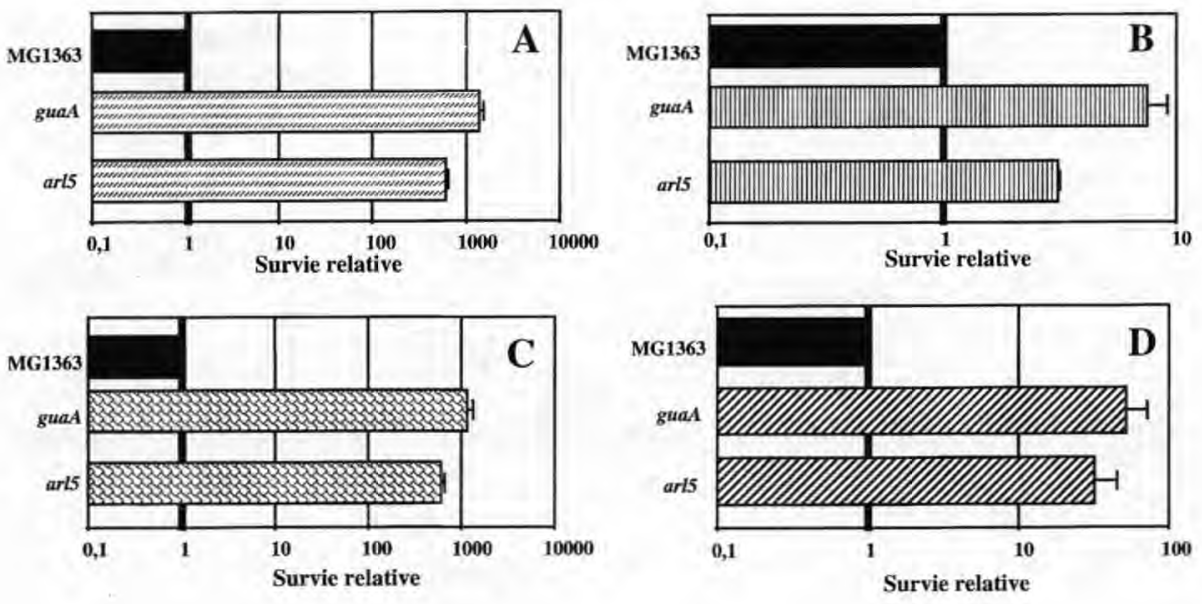

Figure 3. Phénotype des mutants de la classe 3. A. Résistance au stress acide. Le test et le calcul des survies relatives des souches sauvage et mutantes ont été réalisés comme décrit dans la légende de la figure 2A. B. Résistance au choc thermique. Le choc thermique est réalisé par transfert des cultures de $30^{\circ} \mathrm{C}$ à $55^{\circ} \mathrm{C}$ pendant $5 \mathrm{~min}$. Le calcul des survies relatives des souches sauvage et mutantes a été réalisé comme décrit dans la légende de la figure $2 \mathrm{~B}$. C et D. Survie en condition de carence en sucre à $\mathrm{pH}$ acide $(\mathbf{C})$ ou à $\mathrm{pH}$ neutre (D). Les cultures sont réalisées en M17 dépourvu de $\beta$ glycérophosphate mais tamponné avec du MOPS à $40 \mathrm{mmol} / \mathrm{L}$ (C) ou à 200 $\mathrm{mmol} / \mathrm{L}$ (D) et complémenté en glucose à $0,5 \%$ (C) ou $0,25 \%$ (D). La survie est mesurée par étalement sur M17 à l'entrée en phase stationnaire et après 2 jours (C) ou 4 jours (D) d'incubation à $30^{\circ} \mathrm{C}$. Les survies relatives sont calculées comme décrit précédemment (légende de la figure 2 ).

Figure 3. Phenotype of class 3 mutants. A. Resistance to acid stress. The challenge conditions and calculation of relative survival of the mutated and wild-type strains are described in figure $2 \mathrm{~A}$. B. Resistance to heat-shock. The headshock was performed by transferring cultures from $30^{\circ} \mathrm{C}$ to $55^{\circ} \mathrm{C}$ for $5 \mathrm{~min}$. The calculation of the relative survival of the mutated and wild-type strains are described in figure 2B. C and D. Survival to carbohydrate starvation at acid (C) or neutral pH (D). Cultures grew to saturation in modified M17 medium: $\beta$-glycerophosphate was replaced by MOPS at $40 \mathrm{mmol} / \mathrm{L}$ (C) or $200 \mathrm{mmol} / \mathrm{L}$ (D) and supplemented with glucose $0.5 \%$ (C) or $0.3 \%$ (D). Survival was measured by plating on M17 after 2 days (C) or 4 days (D) of incubation at $30^{\circ} \mathrm{C}$. The relative survival under different conditions was calculated as described in figure 2.

arl5 ne présente pas d'homologie significative avec les banques de données. Les autres mutants sont déficients dans les gènes hpt (hypoxanthine-guanine phosphoribosyl transférase [10]), guaA (GMP synthétase [8]) et relA (pppGpp synthétaseI [9]). Ces trois gènes sont impliqués dans la voie de biosynthèse des purines et du (p)ppGpp que nous désignerons dans ce texte sous l'appellation GP (figure 4) [13]. Cette étude suggère que chez $L$. lactis, le taux intracellulaire de GP pourrait être un signal ou un effecteur de stress.
D'autres études réalisées aussi bien sur des bactéries $\mathrm{Gram}^{+}$que Gram ${ }^{-}$impliquent les GP comme messagers intracellulaires de stress. Ainsi, chez E. coli, le (p)ppGpp est un des régulateurs du facteur sigma-S, impliqué dans la résistance globale aux stress induite en phase stationnaire [5]. Chez B. subtilis, des variations du taux de GTP induirait la sporulation [6]. Nous proposons que chez $L$. lactis, le taux de GP intracellulaire soit un signal de stress contrôlant l'expression d'un mécanisme de résistance globale aux stress. 


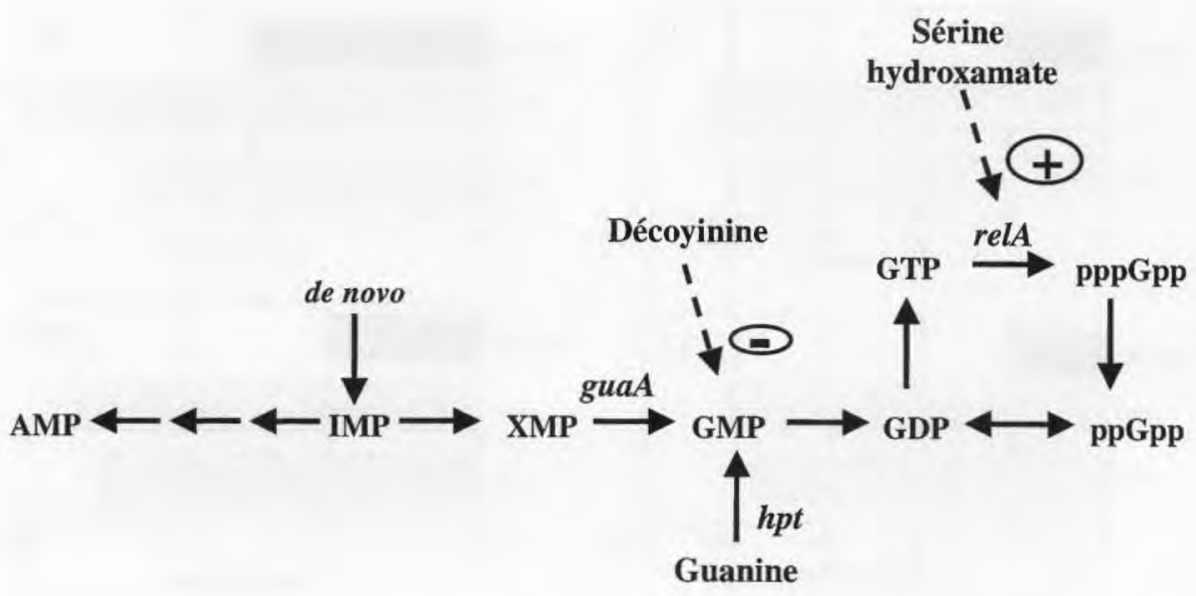

Figure 4. Représentation schématique de la voie de biosynthèse des GP. Les gènes pour lesquels nous avons obtenu des mutants sont indiqués en italique. Les actions de la décoyine et la sérine hydroxamate sont schématisées par des flèches.

Figure 4. Schematic representation of the biosynthesis pathway of GP. The genes for which we obtained mutants are indicated in italics. The effects of decoyinine and serine hydroxamate are symbolized by arrows.

\subsection{Rôle régulateur des taux de GP intracellulaire}

Afin de tester le rôle régulateur des variations des taux de GP intracellulaire, nous avons traité la souche sauvage, MG1363, avec différentes molécules affectant les taux de GTP/(p)ppGpp et examiné sa survie à un stress acide létal. La survie de la souche a été mesurée en condition acide avec et sans traitement. La décoyinine, inhibiteur du produit du gène guaA, la GMP synthétase, et la sérine hydroxamate, inducteur de la réponse stringente et de la synthèse de (p)ppGpp, augmentent la résistance au stress acide de MG1363 d'un facteur 100 par rapport à l'échantillon non traité (résultat non montré). Ce résultat établit l'importance des taux intracellulaires de GP dans l'acquisition d'une résistance au stress acide et, comme le suggèrent nos résultats, dans l'acquisition d'une résistance globale au stress.

\section{CONCLUSION}

Cette étude démontre qu'une seule mutation peut conférer à une souche de L. lactis un avantage considérable en terme de survie à un ou à plusieurs stress. Le système de transposition utilisé dans ce travail permet à la fois d'identifier précisément le gène muté (par séquençage) et d'obtenir des mutants alimentaires. En effet, ces mutants ne comportent aucun $\mathrm{ADN}$ étranger puisque seule une séquence d'insertion originaire de lactocoque (ISS1, [3]) est responsable de la mutation. L'intérêt d'identifier précisément la mutation outre la compréhension des mécanismes bactériens de résistance aux stress est i) de pouvoir utiliser cette mutation comme marqueur de souche et ii) d'être capable de reproduire cette mutation (et donc le phénotype de résistance) dans d'autres souches de lactocoques, voire dans d'autres bactéries lactiques. 
L'étude phénotypique montre qu'il existe chez L. lactis de nombreux recoupements entre les différentes réponses aux stress. En effet, plusieurs des mutants sélectionnés en condition acide s'avèrent aussi plus résistants que la souche sauvage au stress oxydant (pstS, arll) ou à de multiples stress (guaA, hpt, relA et arl5). Nous montrons que les pools intracellulaires de GP auraient chez $L$. lactis un rôle de messager intracellulaire dirigeant l'induction d'une résistance globale aux stress.

Nous pensons que ce type d'approche et ces mutants pourraient s'avérer utiles dans les procédés industriels impliquant les bactéries lactiques. En effet, ces mutants alimentaires plus performants que la souche sauvage pour survivre à de brusques variations de l'environnement pourraient i) améliorer le rendement et la reproductibilité des productions, ii) mieux survivre au stockage facilitant la conservation des ferments et le redémarrage des fermentations et iii) mieux résister, chez l'homme, au transit intestinal, permettant d'obtenir des effets probiotiques plus marqués.

\section{REMERCIEMENTS}

Nous remercions S. Sourice pour sa participation au séquençage, P. Duwat, P. Serror et M. El Karoui pour les discussions concernant ce travail, S. D. Ehrlich pour son soutien.

\section{RÉFÉRENCES}

[1] Bearso S., Bearson B., Foster J.W., Acid stress responses in enterobacteria, FEMS Microbiol. Lett. 147 (1997) 173-180.

[2] Hartke A., Bouché S., Giard J.C., Benachour A., Boutibonnes P., Auffray Y., The lactic acid stress response of Lactococcus lactis subsp. lactis, Curr. Microbiol. 33 (1996) 194-199.

[3] Huang D.C., Novel M., Huang X.F., Novel G., Nonidentity between plasmid and chromosomal copies of ISSI-like sequences in Lac- tococcus lactis subsp. lactis CNRZ270 and their possible role in chromosomal integration of plasmid, Gene 118 (1992) 39-46.

[4] Kaneko T,, Sato S, Kotani H., Tanaka A., Asamizu E., Nakamura Y., Miyajima N., Hirosawa M., Sugiura M., Sasamoto S., Kimura T., Hosouchi T., Matsuno A., Muraki A., Nakazaki N., Naruo K., Okumura S., Shimpo S., Takeuchi C., Wada T., Watanabe A., Yamada M.. Yasuda M,, Tabata S., Sequence analysis of the genome of the unicellular cyanobacterium Synechocystis sp. strain PCC 6803. II. Sequence determination of the entire genome and assignement of potential protein-coding regions, DNA Res. 3 (1996) 109-136.

[5] Lange R., Fisher D., Hengge-Aronis R., Identification of transcriptional start sites and the role of $\mathrm{ppGpp}$ in the expression of $r p o S$, the structural gene for the $\sigma^{5}$ subunit of RNA polymerase in Escherichia coli, J. Bacteriol. 177 (1995) 4676-4680.

[6] Lopez J.M., Dromerick A., Freese E., Response of guanosine 5' -triphosphate concentration to nutritional changes and its significance for Bacillus subtilis sporulation, J. Bacteriol. 146 (1981) 605-613.

[7] Maguin E., Prevost H., Ehrlich S.D., Gruss A., Efficient insertional mutagenesis in lactococci and other gram-positive bacteria, J. Bacteriol. 178 (1996) 931-935.

[8] Mantsala P., Zalkin H., Cloning and sequence of Bacillus subtilis purA and guaA, involved in the conversion of IMP to AMP and GMP. J. Bacteriol. 174 (1992) 1883-1890.

[9] Mechold U., Steiner K., Vettermann S., Malke H., Genetic organization of the streptokinase region of the Streptococcus equisimilis H46A chromosome, Mol Gen Genet 241 (1993) 129-140.

[10] Nilsson D. Lauridsen A.A., Isolation of purine auxotrophic mutants of Lactococcus lactis and characterization of the gene hpt encoding hypoxanthine guanine phosphoribosyltransferase, Mol. Gen. Genet. 235 (1992) 359-364.

[11] Rallu F., Gruss A., Maguin E., Lactococcus lactis and stress, Antonie Van Leeuwenhoek 70 (1996) 243-251.

[12] Takemaru K., Mizuno M., Kobayashi Y., A Bacillus subtilis gene cluster similar to the Escherichia coli phosphate-specific transport (pst) operon: evidence for a tandemly arranged pst $B$ gene, Microbiology 142 (1996) 2017-2020.

[13] Zalkin H., De novo purine nucleotide synthesis. In: Sonenshein A., Hoch J.A., Losick R. (Eds), Bacillus subtilis and other gram-positive bacteria, ASM Press, Washington DC, 1993. 